\title{
INTERNAL ROTATION WITH A LOW THREEFOLD BARRIER; COMPARISON BETWEEN DIFFERENT BASIS FUNCTIONS*
}

\author{
V.M. STOLWIJK and B.P. VAN EIJCK \\ Department of Structural Chemistry, University of Utrecht, Padualaan 8, 3584 CH Utrecht \\ (The Netherlands)
}

(Received 26 April 1988)

\begin{abstract}
For the treatment of the effects of a $C_{3 \mathrm{v}}$ internal rotor on the rotational spectrum of a molecule we have compared two different sets of basis functions, i.e. Mathieu functions and free rotor functions. In both cases the internal axes system was used. 'The behaviour of two model substances, based on the geometries of 1-chloro-2-butyne and meta-fluorotoluene, was studied for values of $V_{3}$ up to $100 \mathrm{~cm}^{-1}$. It was found that for this range of barriers the use of basis functions from more than one torsional state is often necessary for a good description of the energy levels. The size of the matrix to be diagonalized depends on the barrier height and the molecular geometry, but is often larger for free rotor functions than for Mathieu functions. Thus for low barrier problems the use of the latter type of functions, which have not often been used in that region, should be more seriously considered.
\end{abstract}

\section{INTRODUCTION}

In molecules with a symmetric internal rotor (e.g., a methyl group) the internal rotation of this group is coupled with the overall rotation of the entire molecule. Although the internal rotation is also coupled with other vibrations, the basic model to describe the system is a rigid rotor attached to a rigid frame. The theory was reviewed by Lin and Swalen [1] almost thirty years ago; chapters in textbooks written by Wollrab [2] and, more recently, by Gordy and Cook [3] are also devoted to it.

Methods to find the energy levels differ in the choice of axes system and basis functions to set up the Hamiltonian matrix. Dealing with a very low barrier to internal rotation $\left(V_{3}<30 \mathrm{~cm}^{-1}\right)$ two methods to describe the internal rotation are the Internal Axis Method (IAM) and the Free Rotor Method (FRM). The basic difference between the two methods is that the potential energy for the internal rotation is included in the torsional equation in the former method, leading to Mathieu functions, whereas the latter method using

*Dedicated to the memory of Professor Walter Gordy. 
free rotor functions treats the potential energy as a perturbation. Thus the FRM should work best for low barriers and the IAM for high barriers, but the border region between the two methods is not well investigated. Both Lin and Swalen [1] and Gordy and Cook [3] advocate the use of free rotor functions when the reduced barrier height, $s$, is less than 2, whereas Wollrab [2] suggests a criterion of several hundred cal mol ${ }^{-1}$ for the barrier height $V_{3}$ (usually corresponding to $s<10$ ). For higher barriers Mathieu functions should be used.

Most of the very few publications where such a low threefold barrier has been studied in asymmetric molecules used the free rotor wave functions as a basis for the Hamiltonian, because the microwave spectrum showed the characteristic features of free internal rotation [4-7]. The sometimes considerable differences between observed and calculated frequencies have been ascribed to interactions of the methyl torsion with the other molecular vibrations $[5,7]$. Such interactions have been studied in more detail by Koput [8,9]. A disadvantage of using free rotor functions is the necessity of diagonalizing huge matrices. For example, to calculate the energy levels of $\mathrm{CH}_{3} \mathrm{ONO}$ [7] nine torsional functions were needed, leading to an $81 \times 81$ matrix for the levels of $J=4$. For larger values of $J$ such calculations tend to become tedious.

The IAM is computationally more efficient. For low-barrier molecules it has only been applied to acetamide [10] and 1-chloro-2-butyne [11]. Acetamide, too, appeared not to satisfy the rigid frame-rigid top model, but for 1-chloro2-butyne a good fit to the observed frequencies was possible with the IAM. Trial calculations for meta-fluorotoluene showed that the IAM could reproduce the observed spectrum equally as well as the FRM used in the literature. These encouraging results gave us some confidence that the IAM can be used for the internal rotation problem, even when the barrier height is very low.

In this paper we endeavour to give some guidelines for the best choice of basis functions in relation to the barrier height and the molecular structure. For two model structures with different asymmetry parameter $\kappa$ the performance of the methods was studied as a function of the barrier height $V_{3}$. The geometrical parameters were taken from meta-fluorotoluene and from 1-chloro2-butyne; in the real molecules the barrier heights are $V_{3}=16.46 \mathrm{~cm}^{-1}[6]$ and $V_{3}=10.05 \mathrm{~cm}^{-1}[11]$, respectively.

\section{THEORY}

The theory of internal rotation is well-known and described thoroughly in papers and textbooks [1-3,12]. For convenience some of it is given here, limited to the case of a rigid frame and one rigid symmetric internal rotor with a threefold barrier, whereas the molecule as a whole has a plane of symmetry. The torsional-rotational energy levels of such a molecule can be calculated using the Hamiltonian: 
$\mathscr{H}=A P_{a}^{2}+B P_{b}^{2}+C P_{c}^{2}+F\left(\rho_{a} P_{a}+\rho_{b} P_{b}-p\right)^{2}+V(\alpha)$

$p=-i \hbar(\partial / \partial \alpha), F=\hbar^{2} / 2 r I_{\alpha}$

$\rho_{g}=\lambda_{g} I_{\alpha} / I_{g} ; \quad r=1-\lambda_{a}^{2} I_{\alpha} / I_{a}-\lambda_{b}^{2} I_{\alpha} / I_{b}$

$A, B, C$ are the rotational constants defined in the principal axis system. $\lambda_{g}$ is the direction cosinus of the internal rotation axis with respect to the $g$-axis of the principal axis system. In this case $\lambda_{c}=0$ (the molecule has a plane of symmetry). $I_{\alpha}$ is the inertial moment of the internal rotor with respect to the internal rotation axis. $V(\alpha)$ is the internal rotation potential.

The potential function used for a symmetric threefold barrier is:

$V(\alpha)=\frac{1}{2} V_{3}(1-\cos 3 \alpha)+\frac{1}{2} V_{6}(1-\cos 6 \alpha)+\ldots$

In most situations the $V_{3}$-term is sufficient in a first approximation.

The energy operator belongs to the $D_{3}$ symmetry group with the degenerate species $E$ and the non-degenerate species $A_{1}$ and $A_{2}$. The Hamiltonian (1) is defined in the principal axis system, and has been used for both choices of basis functions to be described below. However, in our opinion it is always advantageous to transform to the "internal axis system". This is defined by the axes $(x, y, z)$, with $x=c$ and $z$ coincident with the vector $\rho$. In the internal axis system the Hamiltonian is:

$$
\begin{aligned}
& \mathscr{H}=R_{z} P_{z}^{2}+R_{y} P_{y}^{2}+R_{x} P_{x}^{2}-2 F \rho P_{z} p+(B-A)\left(\rho_{a} \rho_{b} / \rho^{2}\right)\left(P_{z} P_{y}\right. \\
& \left.+P_{y} P_{z}\right)+F p^{2}+V(\alpha) \\
& R_{z}=\left(\rho_{a}^{2} \mathrm{~A}+\rho_{b}^{2} \mathrm{~B}+\mathrm{F} \rho^{4}\right) / \rho^{2} ; \rho=\left(\rho_{a}^{2}+\rho_{b}^{2}\right)^{\frac{1}{2}} \\
& R_{y}=\left(\rho_{a}^{2} \mathrm{~B}+\rho_{b}^{2} \mathrm{~A}\right) / \rho^{2} ; \quad \mathrm{R}_{x}=\mathrm{C}
\end{aligned}
$$

The basis functions to be used in forming the energy matrix are:

$\Psi_{J K M \tau}=\mathrm{e}^{i K \chi} \cdot S_{J K M}(\theta, \phi) \cdot \phi_{\tau}(\alpha) \equiv \Phi_{J K M} \cdot \phi_{\tau}(\alpha)$

The functions $\Phi_{J K M}$ are the orthonormal eigenfunctions of a rigid symmetric rotor and the $\phi_{\tau}(\alpha)$ are the torsional functions. Different torsional functions are recommended [1] in relation to the reduced barrier height $s$.

Intermediate barrier height ( $\mathrm{s}>2$ )

In this case the eigenfunctions of the Mathieu torsional equation

$\left[F p^{2}-2 F \rho K p+V(\alpha)\right] \cdot \phi_{K v \sigma}(\alpha)=E_{K v \sigma} \cdot \phi_{K v \sigma}(\alpha)$

can be used. Here $A$ and $E$ symmetry are denoted by $\sigma=0$ and $\sigma= \pm 1$, respectively. These normalized eigenfunctions $\phi_{K v \sigma}(\alpha)$ are orthogonal neither in the rotational quantum number $K$ nor in the torsional quantum number $v$. The 
advantage is that matrix elements connecting different $v$ values diminish with increasing barrier height, and can often be neglected or reduced in order of magnitude by a Van Vleck transformation. Traditionally, this method using Mathieu functions and a Van Vleck transformation is called IAM and an extensive exposition can be found elsewhere [1-3,12]. When the conditions for using the Van Vleck transformation are not fulfilled, a larger matrix must be diagonalized. This method will be referred to as MFMv (Mathieu Function Method; $v$ represents the torsional quantum number of the highest torsional level used to make up the energy matrix). For the ground vibrational state the IAM is equivalent to MFM0; for excited states the IAM uses the corresponding block out of the more complete matrix used in the MFM. In this context the name IAM is somewhat confusing, because in this paper the MFM and the FRM also have the internal axis system as starting point for the calculations of the energy levels.

\section{Low barrier height $(\mathrm{s}<2)$}

In this case the normalized free rotor functions

$\phi_{m}(\alpha)=(2 \pi)^{-\frac{1}{2}} \mathrm{e}^{i m \alpha}$

seem to be the most suitable. Here $m$ is the torsional quantum number. The relation betwen the quantum numbers $v$ and $m$ can be deduced from Fig. 1 ( $v$ and $m$ are only good quantum numbers for the limiting cases of infinite and zero barrier height, respectively).

In the FRM the energy matrix consists of the following non-zero elements:

$$
\begin{aligned}
& \langle J K M m|H| J K M m\rangle \\
& \qquad=R_{z} K^{2}+\frac{1}{2}\left(R_{y}+R_{x}\right)\left[J(J+1)-K^{2}\right]-2 F \rho K m+F m^{2}+\frac{1}{2} \sum_{n} V_{n} \\
& \langle J K M m|H| J, K \pm 1, M m\rangle \\
& \quad=(B-A)\left(\rho_{x} \rho_{b} / \rho^{2}\right)\left[\frac{1}{2}(2 K \pm 1)\right][J(J+1)-K(K \pm 1)]^{\frac{1}{2}} \\
& \langle J K M m|H| J, K \pm 2, M m\rangle \\
& \quad=\frac{1}{4}\left(R_{y}-R_{x}\right)[J(J+1)-K(K \pm 1)]^{\frac{1}{2}}[J(J+1)-(K \pm 1)(K \pm 2)]^{\frac{1}{3}}
\end{aligned}
$$

A term $\cos (n \alpha)$ in the potential function causes off-diagonal elements in $m$, because

$\left\langle J K M m|\cos n \alpha| J K^{\prime} M, m \pm n\right\rangle=\frac{1}{2} \delta_{K, K^{\prime}}$

leading to an infinite energy matrix. From considerations of symmetry of the Harialtonian the matrix can be factorized in non-degenerate $A$ and degenerate $E$ levels with $m=3 k \pm \sigma(k=0, \pm 1, \pm 2 \ldots$ ) (Fig. 2$)$. The infinite matrix must be truncated after a reasonable expansion in $m$. The influence of the discarded 


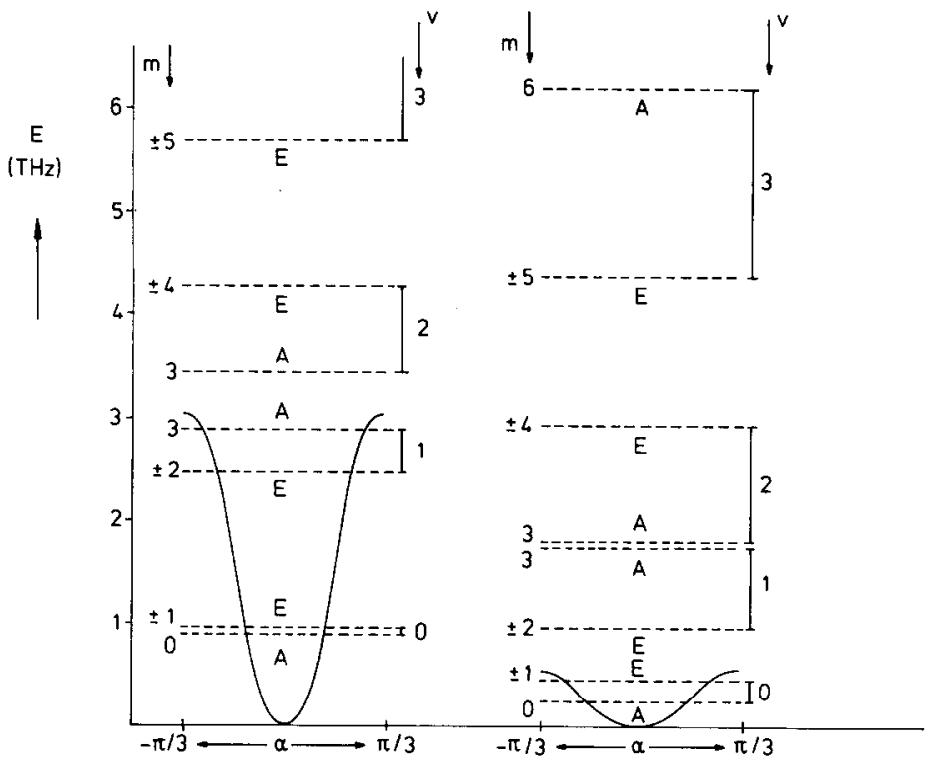

Fig. 1. Torsional rotational energy levels $(J=0)$ of $\mathrm{FC}_{6} \mathrm{H}_{4} \mathrm{CH}_{3}$. The levels are labeled with the torsional quantum number $m$ or $v$ and the symmetry symbol $A$ or $E$. Left, $V_{3}=100 \mathrm{~cm}^{-1}$; right, $V_{3}=16.46 \mathrm{~cm}^{-1}$.
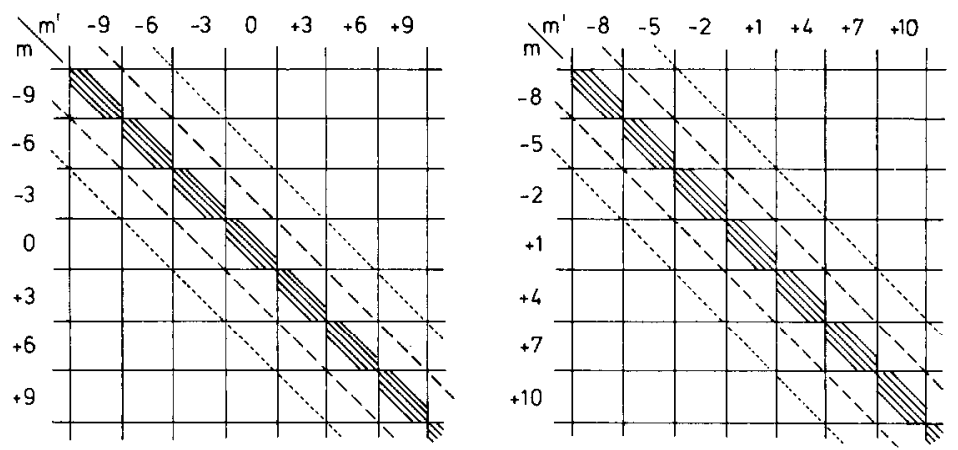

Fig. 2. The infinite energy matrix given by eqn. (7) and eqn. (8). In every square $K$ goes from $-J$ to $+J$. Left: the non-degenerate levels with symmetry $A_{1}$ resp. $A_{2}$. Right: the degenerate levels with $E$ symmetry. $\equiv$ Eqn. (7); - - - $-\frac{1}{4} V_{3} ; \cdots \cdots-\frac{1}{4} V_{6}$.

basis functions (denoted by $m^{\prime}$ ) can be approximated by a Van Vleck transformation. For each $m^{\prime}$ a contribution

$\frac{V_{n}^{2}}{16 F\left(m^{\prime}-m\right)\left(2 \rho K-m^{\prime}-m\right)}$, with $n=\left|m^{\prime}-m\right|$

has to be added to the diagonal elements $\langle J K M m|H| J K M m\rangle$. In this paper the method applying free rotor functions, including the Van Vleck transformation, will be called FRMn ( $n$ representing the number of free rotor functions used). 
From eqn. (7) it is easy to see that only the diagonal elements are dependent on $m$ and that the blocks $+m$ and $-m$ are equal, leading to degenerate energy levels (except for $m=0$ ) in the case of completely free internal rotation. For a non-zero barrier the degeneracy between the blocks $m=+3$ and $m=-3$ is lifted directly by a $V_{6}$ term and indirectly by a $V_{3}$ term, leading to effects of comparable magnitude [12]. Thus here the FRM matrix must contain at least the blocks $m=-3,0,+3$.

\section{CALCULATIONS}

Both the IAM and MFM computer programs, written in Algol60, have been described earlier [13,14]. For the FRM calculations a Fortran77 program has been written that diagonalizes the $A$ and $E$ energy matrix separately (Fig. 2) with any number of free rotor functions. Transition frequencies and line intensities can also be calculated.

Unfortunately the computational costs of the MFM and the FRM could not be compared realistically in this work. Apart from the difference in program language the programs do not use the same diagonalization procedure. For large values of $J$ the order of the matrix will be critical in the calculation; this order is $(v+1)[2 J+1]$ for MFMv and $n[2 J+1]$ for FRM $n$.

\section{TABLE 1}

Molecular parameters for model calculations

\begin{tabular}{lcc}
\hline & $\mathrm{C}_{4} \mathrm{H}_{5} \mathrm{Cl}[1]$ & $\mathrm{FC}_{6} \mathrm{H}_{4} \mathrm{CH}_{3}[2]$ \\
\hline$A(\mathrm{MHz})$ & 16039.94 & 3654.071 \\
$B(\mathrm{MHz})$ & 1489.03 & 1762.099 \\
$C(\mathrm{MHz})$ & 1385.03 & 1197.584 \\
& & \\
$\kappa^{\mathrm{a}}$ & -0.9858 & -0.5404 \\
$F(\mathrm{MHz})$ & 172525.520 & 164204.000 \\
$I_{\alpha}\left(\mathrm{u} . \AA^{2}\right)$ & 3.2258 & 3.14 (ass) \\
$\theta^{\mathrm{b}}\left({ }^{\circ}\right)$ & 19.5995 & 29.627 \\
& & \\
$\rho_{a}$ & 0.09645174 & 0.01973493 \\
$\rho_{b}$ & 0.00318826 & 0.00541257 \\
$\rho$ & 0.0965044 & 0.0204637 \\
$R_{x}(\mathrm{MHz})$ & & \\
$R_{y}(\mathrm{MHz})$ & 1385.030 & 1197.585 \\
$R_{z}(\mathrm{MHz})$ & 1504.912 & 1894.458 \\
\hline
\end{tabular}

${ }^{a} \kappa=(2 B-A-C) /(A-C)$.

${ }^{\mathrm{b}} \theta$ The angle between the $a$ principal axis and the internal rotation axis.

[1]: From ref. 11.

[2]: Calculated from data given in ref. 6 , see text. 
Calculations were made for energy levels of the molecules 1-chloro-2-butyne $\left(\mathrm{C}_{4} \mathrm{H}_{5} \mathrm{Cl}\right)$ and meta-fluorotoluene $\left(\mathrm{FC}_{6} \mathrm{H}_{4} \mathrm{CH}_{3}\right)$ for different values of the barrier height. The molecular parameters used in the calculations are given in Table 1 . The rotational constants and $\theta$ of meta-fluorotoluene were obtained by an IAM refinement (the root mean square deviation being $0.5 \mathrm{MHz}$ ) of the ground torsional state, $v=0$, with the transition frequencies of $m=0$ and $m=1$ reported in ref. 6 . In the calculations no centrifugal distortion was taken into account and $V_{6}$ was set to zero.

The energy levels calculated with the different methods were compared for torsional states up to and including $m=4(v=2)$ and $J=6$. The differences increase with $J$ and for a certain $J$ mostly with $K_{-1}$, so the results refer to the maximum values for $J=6$. Trial calculations for $J=10$ confirm qualitatively the observations reported below.

\section{RESULTS AND DISCUSSION}

When enough basis functions are taken into account, the energy levels calculated with the MFM and FRM are identical for both 1-chloro-2-butyne and meta-fluorotoluene for values of the barrier height up to $V_{3}=25 \mathrm{~cm}^{-1}$. (There is no reason to believe that beyond this barrier height the methods would diverge.) These data indicate that the methods and programs are correct and serve as a standard to evaluate the usefulness of smaller basis sets.

The number of basis functions needed in the FRM to set up an adequate energy matrix depends on the barrier height and the vibrational level. The quality of the results of calculations FRM1, FRM3, and FRM5 is shown in Fig. 3 . The molecules behave identically, so only the results on 1-chloro-2-butyne are shown. This figure is the most relevant part of a more complete one with ordinate values from $10^{-3}$ to $10^{+7}$.

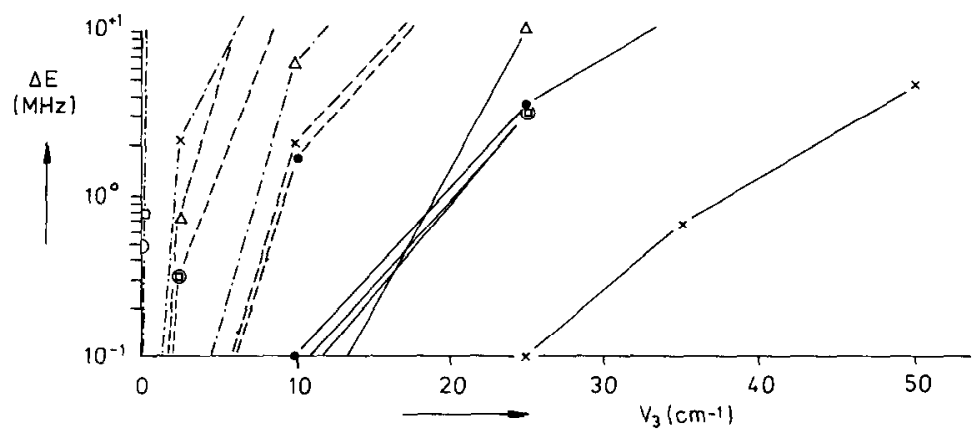

Fig. 3. The influence of the number of torsional functions on the value of the calculated energy levels. $\Delta E$, the maximum difference between the correct energy levels $(J=6)$ and the same levels calculated by FRM $n(n=1,3,5), \ldots . . .$, FRM1; ---, FRM3; - , FRM5; $\times, m=0 ; \square, m=1 ; 0$, $m=2 ; \quad$, $m=3 ; \Delta, m=4$. 

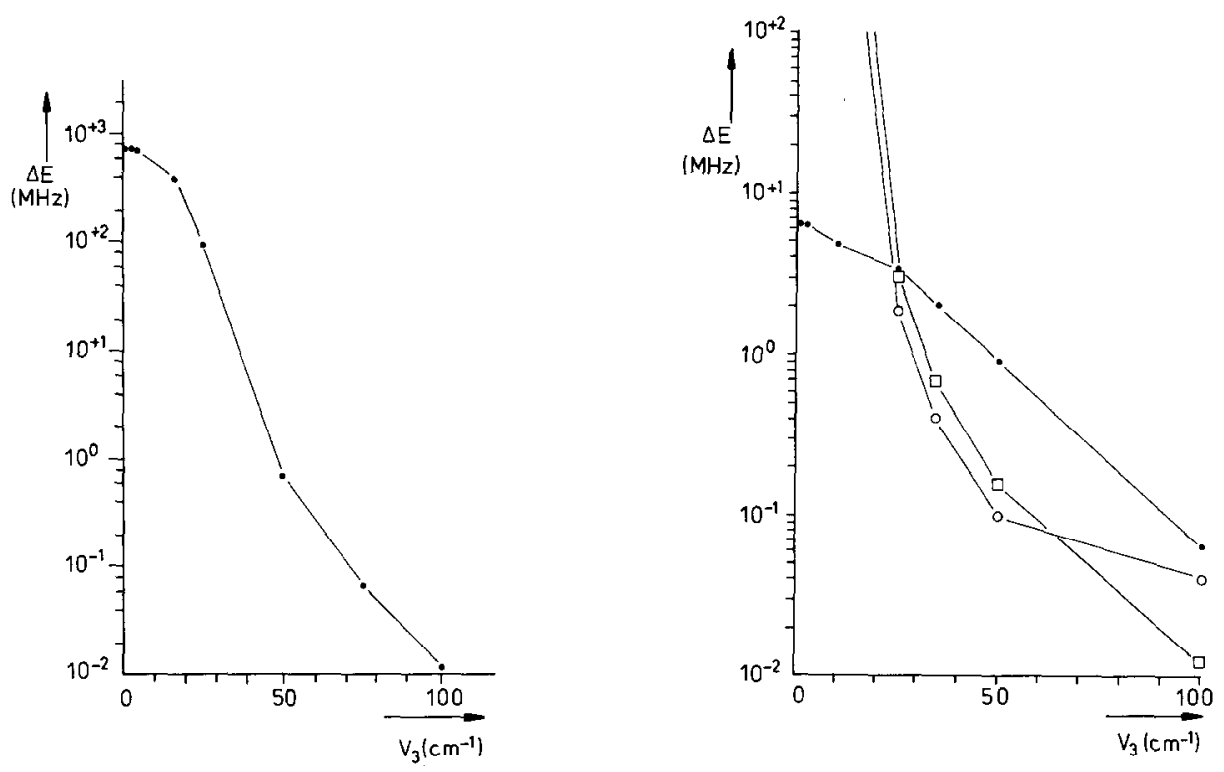

Fig. 4. The maximum difference $\Delta E(\mathrm{MHz})$ between the energy levels $(J=6, m= \pm 3)$ calculated by the IAM and by the MFM/FRM for different barrier heights for meta-fluorotoluene.

Fig. 5. The maximum difference $\Delta E(\mathrm{MHz})$ between the energy levels $(J=6)$ calculated by the IAM and by the MFM/FRM for different torsional levels and barrier heights for 1-chloro-2butyne. $\square, m=1 ; \circ, m=2 ; \bullet, m=3$.

Using Mathieu functions the use of MFMv is generally adequate to reproduce the energy levels up to and including $v-1$. This means that the matrix to be diagonalized is smaller in the MFM than in the FRM.

The behaviour of the two molecules towards the IAM is quite different. For meta-fluorotoluene the torsional energy levels $m=0,1,2$, and 4 calculated with the IAM are correct; for $m= \pm 3$ the deviations depend on the barrier height (Fig. 4). This is not surprising in view of the possible near degeneracy between the $A$ levels of $v=1$ and $v=2$ (Fig. 1). For 1-chloro-2-butyne significant deviations occur for $m=1,2$, and 3 (Fig. 5); for $m=0$ and $m=4$ all errors are less than $0.01 \mathrm{MHz}$ with the exception of the two highest energy levels for $m=4$, $J=6$, where the deviations are suddenly larger than $250 \mathrm{MHz}$. This is outside the bounds of the picture.

For the two molecules studied the IAM performs best for the more asymmetric one. Some additional calculations for models with $\kappa=0$ showed that this conclusion is not generally valid. Our interpretation of the origin of the difference between the two molecules is illustrated in Fig. 6. The energy levels shown in this figure are the lowest and highest of every rotational quantum number $J: J 0 J$ and $J J 0$. The reduced barrier height is the same for both molecules $(s=0.1939)$. From this figure the difference is obvious: the mixing of 


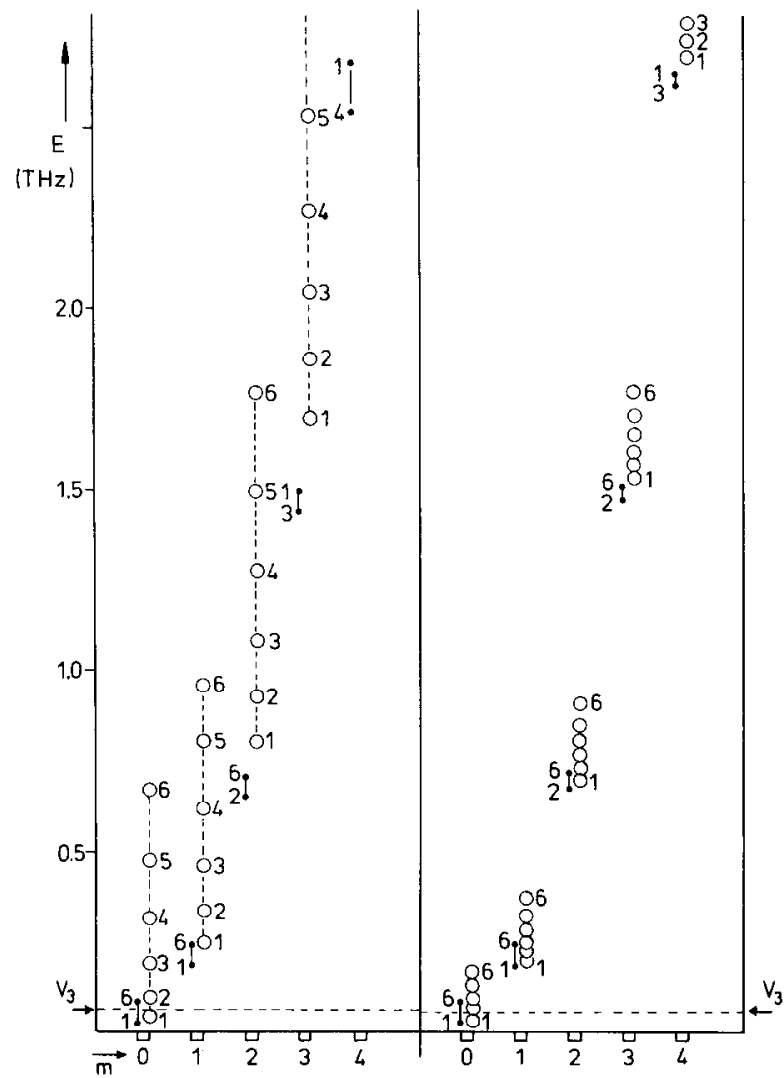

Fig. 6. The torsional-rotational energy levels ( $J 0 J$ and $J J 0)$ of 1-chloro-2-butyne (left) and metafluorotoluene (right) calculated by the IAM for a few torsional states. The reduced barrier height $s$ has the same value for both molecules $(s=0.1939)$. On the scale of this figure no difference can be made between the energy levels of $m=+3$ and $m=-3$. $\bullet$, The energy levels $J 0 J$; $\circ$, the energy levels $J J 0$.

different torsional states of 1-chloro-2-butyne increases with the value of $J$ or, to be more accurate, with the value of $K_{-1}$. Thus the levels $m=0$ and $m=4$ are reasonably isolated from the levels with $m^{\prime}=m \pm 3$, whereas considerable mixing can be expected between $m= \pm 1$ and $m=\mp 2$. The few large deviations for $m= \pm 4, J=6$ are caused by interaction with the state $m=\mp 5$. Along with the general interaction between $m=+3$ and $m=-3$ this accounts for the observations given above (Fig. 5 ). For the molecule in question, where off-diagonal elements in $K$ are small, it turns out that the interaction is negligible for $K_{-1}<2$. Indeed, the observed frequencies for 1-chloro-2-butyne, which mostly satisfied this criterion, could be well reproduced by the IAM [11]. 


\section{CONCLUSIONS}

Free rotor functions and Mathieu functions give an equally good description for low barriers to internal rotation, if the basis sets are complete enough. The size of the complete set of free rotor functions increases with the barrier height; for the Mathieu functions the molecular geometry is a more important factor. In both methods special attention has to be paid to the levels $m= \pm 3$.

Traditionally, free rotor functions are used for very low barriers. Fig. 3 shows that for intermediate barrier heights this method can only be used if one is prepared to diagonalize extremely large matrices when "high" $J(J>10)$ transitions are involved. Stated differently, if FRM3 is computationally feasible, then the barrier should not exceed a few $\mathrm{cm}^{-1}$. With the same matrix order, i.e. $3(2 J+1)$, one can use the MFM2 based on Mathieu functions. Better results can often be obtained in this way, at the expense of greater numerical complexity. After inspection of diagrams such as Fig. 1 and Fig. 6 the optimum basis set can be selected. In favorable cases, such as meta-fluorotoluene, where the torsional states are well separated, the IAM ( $\equiv$ MFM with just one torsional state) is adequate for all but the $m= \pm 3$ torsional states.

\section{REFERENCES}

1 C.C. Lin and J.D. Swalen, Rev. Mod. Phys., 31 (1959) 841.

2 J.E. Wollrab, Rotational Spectra and Molecular Structure, Academic Press, New York, 1977.

3 W. Gordy and R.L. Cook, Microwave Molecular Spectra, Techniques of Chemistry, Vol. XVIII, 3rd edn., Wiley, New York, 1984.

4 V.W. Laurie and D.R. Lide, Jr., J. Chem. Phys., 31 (1959) 939.

5 R.G. Lett and W.H. Flygare, J. Chem. Phys., 47 (1967) 4730.

6 H.D. Rudolph and A. Trinkaus, Z. Naturforsch., Teil A, 23 (1968) 68.

7 P.H. Turner, M.J. Corkill and A.P. Cox, J. Phys. Chem., 83 (1979) 1473.

8 J. Koput, J. Mol. Spectrosc., 106 (1984) 12; 115 (1986) 131.

9 J. Koput, J. Mol. Spectrosc., 118 (1986) 189.

10 T. Kojima, E. Yano, K. Nakagawa and S. Tsunekawa, J. Mol. Spectrosc., 112 (1985) 494; 122 (1987) 408.

11 V.M. Stolwijk and B.P. van Eijck, J. Mol. Spectrosc., 124 (1987) 92.

12 H. Dreizler, Fortschr. Chem. Forsch., 10 (1968) 59.

13 B.P. van Eijck, J.van Opheusden, M.M.M. van Schaik and E. van Zoeren, J. Mol. Spectrosc., 86 (1981) 465.

14 B.P. van Eijck and F.B. van Duijneveldt, J. Mol. Spectrosc., 102 (1983) 273; B.P. van Eijck, A. Dubrulle, J. Demaison and J.L. Ripoll, J. Mol. Spectrosc., 112 (1985) 95. 